\title{
Utilizing rs-fMRI's Mean to Localize Seizure Focus
}

\author{
Byron Bernal, Magno R. Guillen, Kathryn Zamora, Nolan Altman \\ Department of radiology, Miami Children's Hospital, Miami, USA \\ Email: Byron.bernal@mch.com
}

Received 3 March 2015; accepted 8 June 2015; published 11 June 2015

Copyright (C) 2015 by authors and Scientific Research Publishing Inc.

This work is licensed under the Creative Commons Attribution International License (CC BY).

http://creativecommons.org/licenses/by/4.0/

(c) (i) Open Access

\begin{abstract}
Purpose: We present for the first time the utilization of an average of the resting-state time points (rs-Mean) to localize epilepsy focus in a group of pediatric patients with medical refractory epilepsy. Materials and Methods: Twenty nine pediatric patients had drug-resistant epilepsy, and 22 subject controls underwent rs-fMRI. Comparison among group means was performed. Regions of Interest (ROI) derived from the nuclear medicine studies (GS) and the focal hyperintensities yielded by the rs-Mean were converted into ellipsoids. Euclidian Distance (ED) among the isocenters of each ROI pair per patient was found. Matching of ROIs was accepted if the ED was equal or less than $50 \mathrm{~mm}$. Group comparison was performed utilizing two-tail T-test. Statistical significance was accepted with $p$ values $<0.01$. Results: Statistically significant differences were found between the patient and normal control's rs-Mean $(p<0.001, t(49)=5.186$; CI $(95 \%)=[512.73,1161.46])$. The rs-Mean findings matched the GS findings in epilepsy patients in $72.4 \%$ of cases. The rs-Mean was positive matching GS in 6 of $9(66.6 \%)$ MR-non-lesional cases. Conclusion: Our study demonstrates that rs-Mean in intractable epilepsy patients differs from normal subjects, and that it may demonstrate the epileptogenic focus in a significant number of patients.
\end{abstract}

\section{Keywords}

Epilepsy, Seizures, fMRI, Resting-State, SPECT, Intractable Epilepsy

\section{Introduction}

Resting-state fMRI (rs-fMRI) is a type of functional magnetic resonance imaging (fMRI) in which the brain is not engaged in any specific task. Temporal correlations of brain spontaneous oscillations as defined by this method reveal intrinsic connectivity networks. Several intrinsic networks have been described in normal volunteers, utilizing Independent Component Analysis (ICA). These include primary visual, secondary visual, auditory, sen- 
sory, motor, default mode, salience, dorsal parieto-frontal (attention), posterior cingulate, precuneus, language, and anterior default mode [1]-[3].

A volume that averages the rs-fMRI time points may be generated during the processing of ICA. This is what we want to introduce as "the resting-state mean" (rs-Mean).

The rs-Mean volume contains not only the voxel-based mean of BOLD variation (T2*) or intrinsic networks, but also the mean of any other T2 signal, as this sequence is sensitive to the relaxation of the transverse magnetization. Thus, the highest intensities are expected for CSF and the lowest for air.

Despite the fact that we do not have a clear understanding of its origin, we have empirically found in many drug-resistant epilepsy cases that the rs-Mean shows coincident findings with nuclear medicine neuroimages.

The aim of this paper is to report the correlation between rs-Mean findings and the results of ictal SPECT or interictal PET studies in a group of pediatric patients with intractable epilepsy who are being evaluated as candidates for resective surgery. We will also report the findings of rs-Mean in a group of young adult normal volunteers. Illustrative cases will be presented. As far as we know, this is the first report of the usefulness of this technique in the detection of the epilepsy focus.

\section{Materials and Methods}

\subsection{Subjects}

The protocol of a retrospective-prospective study was approved by our IRB. Parents or legal guardians of prospective patients signed a written approved-informed consent. The typical control group and patients demographics are summarized in Table 1(a) and Table 1(b) Fifty patients imaged between the dates of May 2011 and May 2013 with a history of intractable seizures and who had undergone either ictal SPECT or interictal PET and resting-state functional MRI were initially taken from a pool of patients attending our Institution. Twenty one cases were excluded for different reasons. Five of the excluded patients had overt inconsistent findings between SPECT and PET; seven cases were excluded because prior brain surgery as the surgical cavity appears with very high intensity in the rs-Mean and may confound the results; two cases because of cystic encephalomalasia cavities, four due to brain tumor, and two cases of Sturge Webber. One additional case was excluded as there was no agreement on the SPECT readings (see below in methods). The patient group consisted of 29 patients with the diagnosis of cortical dysplasia (15), non-lesional cases (9), tuberous sclerosis (3) and viral encephalitis sequelae (2). All diagnoses were done by MRI criteria. Tuberous sclerosis cases were included despite multiple tubers in those in which the tuber demonstrating frequent epileptogenic activity was spatially concordant with nuclear medicine findings.

The control group consisted of 22 normal right handed subjects (22 - 34 years range). These normal subjects were taken from an online database at the neuroinformatics repository of the Autism Brain Imaging Data Exchange (ABIDE, http://fcon_1000.projects.nitrc.org/indi/abide/). Data sets were all acquired in the same $1.5 \mathrm{~T}$ scanner. All subjects have corresponding structural MRI and demographic information.

\subsection{Neuroimaging}

In the group of patients, when both interictal PET and ictal SPECT imaging were performed, and they were consistent at least at the lobe level, the ictal SPECT was chosen for comparison as its sensitivity has been found higher than 90\% in large partial epilepsy series [4]. Ictal SPECT was performed using a Siemens Multispect 3 gamma camera (Hoffman States, IL). Technetium-HMPAO was used as the radiotracer at a dose of 300 microcuries/kilogram with a minimum dose of 3 milicuries and a maximum dose of 20 milicuries. The patient was monitored by EEG and Video EEG during the exam to determine the onset of seizures for injection. Ictal SPECT were considered valid if the injection of the radiopharmaceutical occurred within 20 seconds after the seizure onset. Interictal PET was performed using a GE Discovery-Dimension ST PET/CT system. FDG was injected at a dose of 140 microcuries/kilogram with a minimum of 1 milicurie and maximum of 15 milicuries. EEG monitoring was utilized to verify interictal condition.

Echo-planar gradient imaging (EPI) BOLD-sensitive sequences were utilized for the Resting State fMRI. The parameters for the EPI sequence acquisition were, 200 timepoints; TR $=2000 \mathrm{~ms}$, TE $=60 \mathrm{~ms}, \mathrm{FA}=90$ degrees, transversal in-plane voxel size $3.75 \times 3.75 \mathrm{~mm}$, field of view (FOV) of $240 \mathrm{~mm}$, resolution $64 \times 64 \times 14$. Axial cuts of $6 \mathrm{~mm}$ with $2 \mathrm{~mm}$ gap were taken in an inferior to superior direction using an 8-channel head-sense coil. 
Table 1. (a) Patient demographics; (b) Normal controls demographics.

(a)

\begin{tabular}{ccc}
\hline & Distribution of patients by gender $(\mathrm{n}=29)$ \\
\cline { 2 - 3 } Attributes & Male & Female \\
Age range & 13 & 16 \\
Age mean/SD & $2-15$ & $2-22$ \\
R Handed & $9.84 / 4.48$ & $9.92 / 5.49$ \\
L Handed & 9 & 11 \\
Ambidextrous & 2 & 3 \\
Unknown Handedness & 1 & 0 \\
L Temporal & 1 & 2 \\
R temporal & 5 & 7 \\
Non-Temporal & 1 & 0 \\
Sedated & 7 & 9 \\
Seaizure to fMRI lag: & 8 & 30.1 \\
Range [Hrs.] & & \\
Maen [Hrs.] & $24-96$ & $0.27 / 72$ \\
\hline
\end{tabular}

(b)

\begin{tabular}{ccc}
\hline & \multicolumn{2}{c}{ Distribution of controls by gender $(\mathrm{n}=22)$} \\
\cline { 2 - 3 } Attributes & Male & Female \\
Gender & 10 & 12 \\
Age range & $23-34$ & $22-34$ \\
Age mean/SD & $9.84 / 3.86$ & $26.8 / 3.65$ \\
R Handed & 8 & 9 \\
L Handed & 1 & 2 \\
Ambidextrous & 1 & 1 \\
\hline
\end{tabular}

To allow co-registration of activation results, an anatomical 3D T1 sequence was also acquired using voxel size $1 \times 256 \times 160$. All patients and control subjects had an axial T1-3D series available.

PET and SPECT interpretation was initially performed by a pediatric neuroradiologist and a clinical report was generated. The same images were blinded reviewed again, as part of this study, by a Radiology fellow (KZ) and abnormal localization of the radiotracer uptake was performed. Only concordant cases were kept in the sample to improve $1 \times 2 \mathrm{~mm}$, resolution $256 \times$ strength of the Standard of comparison. Only one case was rejected on these grounds.

\section{3. fMRI Post-Processing}

The Resting State fMRI data sets were processed using MELODICA version 3.10 from FSL 4.1.9 (http://www.fmrib.ox.ac.uk/). MELODICA yields the rs-Mean in two different files with identical content: called mean and mean_func files. The mean file was taken for the analysis.

Both, patient subjects and typical control-rs-Mean volumes were intensity normalized, smoothed and coregis- 
tered to a "reference anatomical data set", which was a standard brain T1 sequence taken from FSL (MNI152_T1_2mm-Brain), voxel size $2 \times 2 \times 2 \mathrm{~mm}$, spatial resolution $91 \times 109 \times 91$. The intensity normalization process consisted in identifying the local maximum intensity for each dataset, corresponding to CSF signal, and selecting the dataset with the global maximum as the "group maximum intensity reference dataset". Then each voxel in each data set was divided by its corresponding local maxima and multiplied by the group maximum intensity. The coregistration and reslicing of the subject's rs-Mean to the "reference anatomical data set" was performed utilizing FLIRT (from FSL library, Ver.5.5), with 12 degrees of freedom.

\subsection{Statistical Analysis}

The mean and standard deviation (SD) for patients and control group were calculated. All the image statistical calculations were performed using the Multi-image Analysis GUI (Mango), which was developed by the Health Science Center of the University of Texas (http://ric.uthscsa.edu/mango/). Statistical comparisons of means (normal vs. patients) were performed in two ways. The first calculation assumed a statistical universe of $n=22, n=$ 29 (subjects), more sensitive to intensity differences between groups; the second calculation assumed a statistical universe of $n=902,629$ voxels (number of voxels on each mean volume), more sensitive to the variance of distribution of intensities within the volumes. The statistical two tailed T-test was performed utilizing the opensource program PSPP (available at http://www.gnu.org/software/pspp/). Statistical significance threshold was set at $\mathrm{p}$ value equal or less than 0.01 .

\subsection{ROIs and Isocenters Selections}

The PET, SPECT, and rs-Mean volumes were coregistered to the subject's axial T1-3D MRI volume utilizing the AMIDE software (http://amide.sourceforge.net). The rs-Mean volume was color-coded using the predefined available NIH look-up-table. The display of the color image was thresholded by adjusting to $80 \%( \pm 10 \%)$ of the maximum intensities present in the study. The areas of abnormal radiotracer uptake on the SPECT or PET and the area of increased intensity on the rs-Mean were marked with 3D-elipsoides as region of interest (ROI). The Euclidian distance between the coordinates of the center of mass of each ROI, provided by AMIDE, was calculated using the Euclidian Distance (ED) Method computed by MATLAB. Matching between rs-Mean and the standard was accepted if the isocenters were located in the same hemisphere and the ED was equal or less than $50 \mathrm{~mm}$. This distance was selected in agreement with prior publications describing the distance error of BOLD activation related to Interictal Epileptic Discharges [5]. Three demonstrative cases are presented.

\section{Results}

All typical controls (TC) and patient's rs-fMRI yield neural components. rs-Mean image was obtained in all of them for further analysis.

\subsection{Group Analysis and Typical Controls}

Typical control subjects showed a symmetric distribution of intensities between the hemispheres (Figure 1). The highest intensities, as expected, were located in the ventricles and in the proximity of the sylvian and interhemispheric fissures. The parenchyma shows higher intensities in the parahippocampal regions, cingulate gyrus, insula and temporal poles. The prefrontal cortex looks cold. In the SD volume all CSF spaces show high variability. Most importantly, the brain parenchyma shows very low and symmetric values indicating low intersubject variability as well as low inter-subject interhemispheric variability.

\subsection{Group Analysis and Epilepsy Patients}

Similar to TC subjects the epilepsy group-mean showed a symmetric distribution of intensities between the hemispheres (Figure 2), except for the temporal lobes, in which there is a slightly higher intensity in the left side, reflecting the distribution of temporal lobe epilepsy in the sample. The highest intensities, as in the TC group were located in the ventricles and in the proximity of the sylvian and interhemispheric fissures. The gray/white-matter distribution of the intensities follows similar pattern than in TC. The SD volume (not shown) demonstrated significant higher values than the control group (see group differences analysis below). 


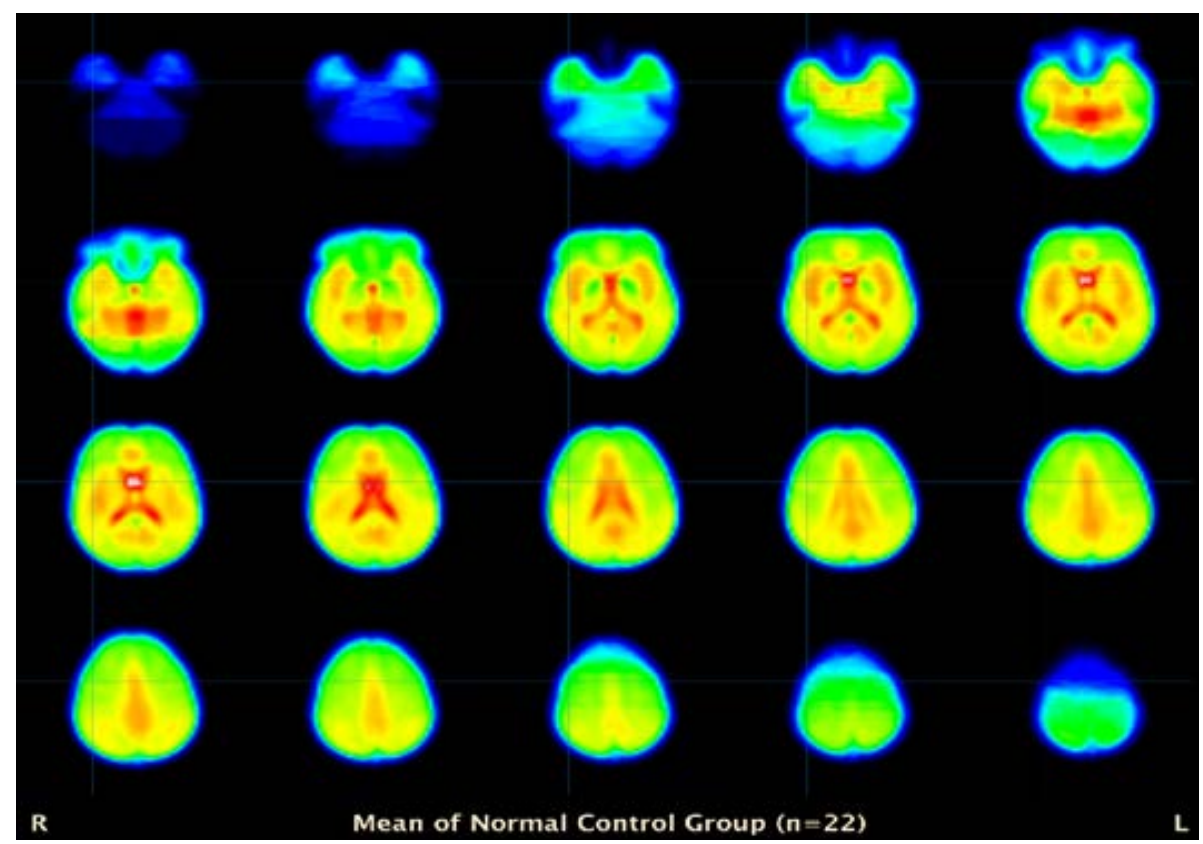

Figure 1. Normalized group-mean for TC. Axial cuts mosaic of the rs-Mean across the normal group. Orientation of insets is in radiological convention. Voxel values are color coded from dark blue (lowest) to white (highest value). Most cephalic and most caudal cuts show the effect of registration that zeroes missing data. Of note is the symmetry of the signal among all homologous areas. Highest values are located in ventricles, sylvian and interhemispheric sulcus. Parenchymal higher signals are located in parietal, occipital and perisylvian areas.

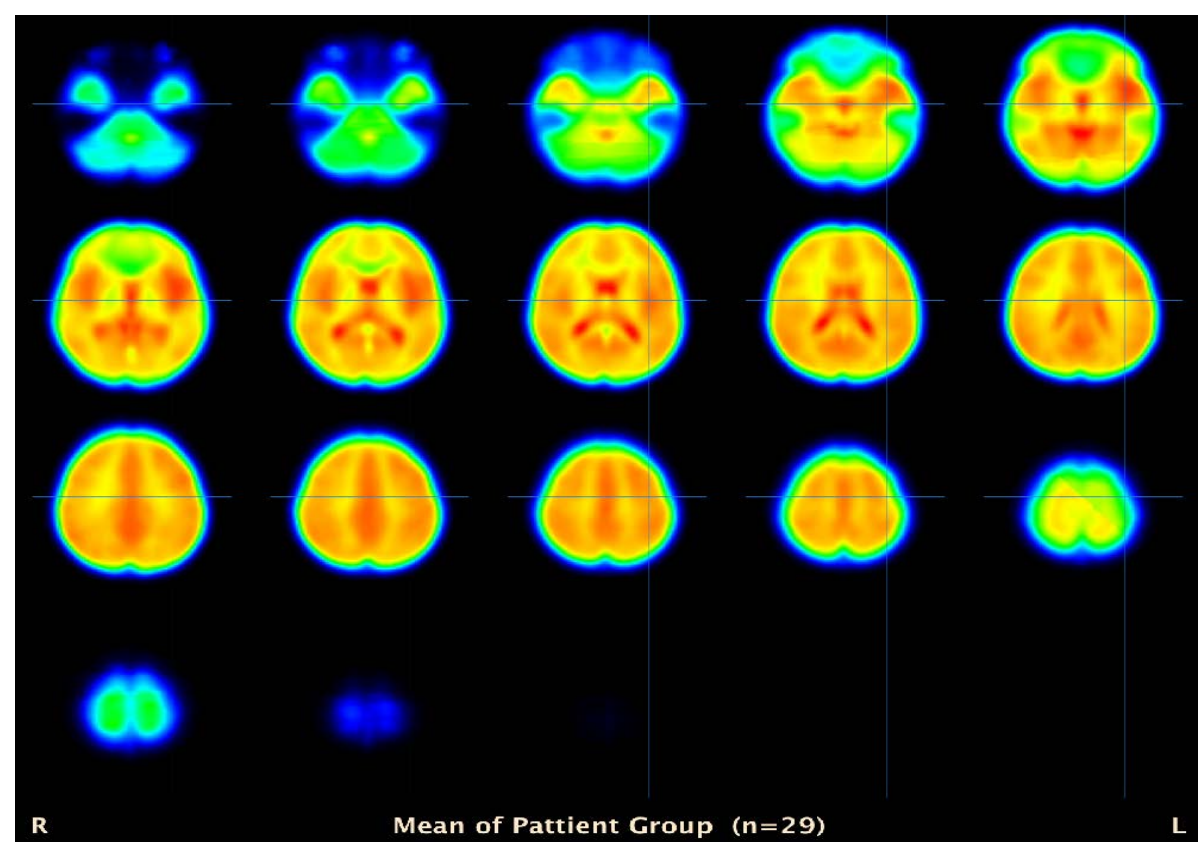

Figure 2. Normalized group mean for patient group. Axial cuts mosaic of the rs-Mean across the Patient group. Orientation of insets is in radiological convention. Voxel values are color coded from dark blue (lowest) to white (highest value). Notice the global higher intensities of cortical areas in this group in comparison to the TC group with maxima kept constant for $\mathrm{CSF}$, an invariant component. The left temporal lobe shows a slightly greater intensity in agreement with the distribution of left and right temporal lobe epilepsy in our sample. 


\subsection{Inter-Group Statistical Comparison}

The mean/SD for the TC group was 4325.81/487.07; the mean/SD of the patient groups was 5162.91/626.45. From these numbers the two-tail t-test based on the universe of number of subjects (N1 $=22$, N2 $=29$ ) we obtained a p < 0.001, t (49) $=5.186$; CI $(95 \%)=[512.73,1161.46])$. The calculations based on the universe of voxels $(\mathrm{N}=902.629$ per volume), yield a $\mathrm{p}<0.001$, for the difference between means. t $(1805257)=1002.24$; CI $(95 \%)=[835.46,838.73]$. These results demonstrated statistically significant difference between the groups for both intensities and intravolume variance.

To present graphically the separation between normal controls and patients, a graph of the mean intensity and total intensity summation of each rs-fMRI mean dataset wasplotted. Figure 3 displays this plot.

\section{4. rs-FMRI vs. Reference Standard Comparison in Patients}

Table 2 presents the list of patients in the study, indicating main demographic variables, the lesion type, lesion location, the time-gap between the last seizure and the rs-Mean, sedation condition, the nuclear medicine Standard, the matching outcome, and the Euclidian distance between the centroids of the ROIs generated using the Standard and the rs-Mean data.

Of the 29 patients included, 21 (72.4\%) of them had imaging that demonstrated a matching overlap in the region of interest between rs-Mean and the Nuclear Medicine Standard. Of these the range of Euclidian Distance was 3.77 - $67.93 \mathrm{~mm}$ (mean 22.65, and SD $16.06 \mathrm{~mm}$ ). With these numbers the estimated error is less than 5 voxels. Of note are the results of the non lesional group. Of the 9 non lesional cases, 6 (66.6\%) showed matching findings between the PET or SPECT and the rs-Mean.

Eight of the 29 patients (27.6\%) demonstrated discordant findings between the selected Standard and the

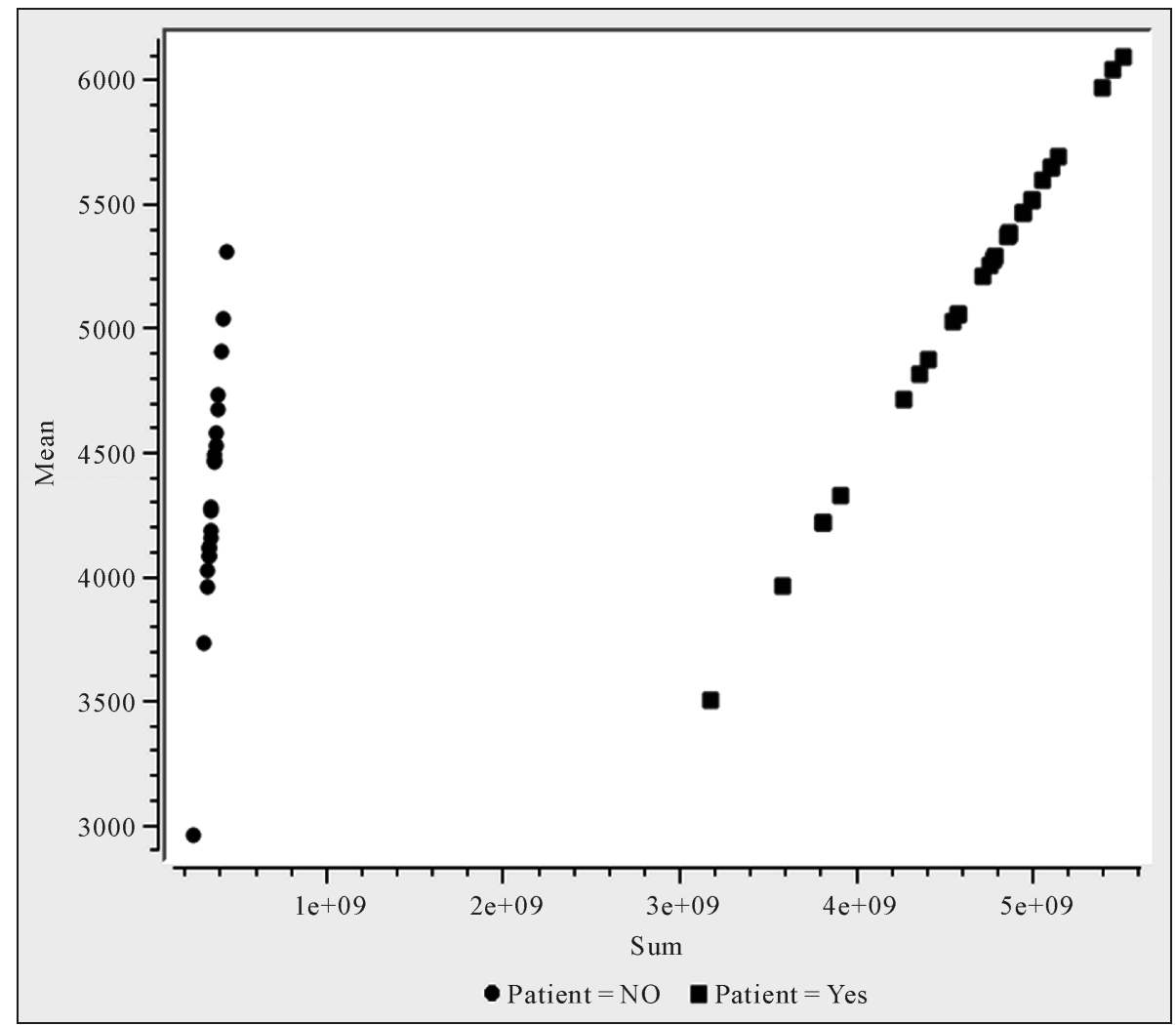

Figure 3. rs-Mean-based group separation. The plot depicts the differences between normal control and patient group members based on their individual means and summation of signal intensities. $\mathrm{Y}$ axis presents the individual mean intensities; $\mathrm{X}$ axis represents the total summation of intensities. Normal control group is represented as a circle while patients are depicted as squares. Note the evident distinction of the groups intensity wise. 
Table 2. Patient specific information and findings.

\begin{tabular}{|c|c|c|c|c|c|c|c|c|c|}
\hline ID & Age & Hand & Sex & MR-Diag & NM Std. & $\mathrm{Gap}^{\mathrm{a}}[\mathrm{Hr}]$ & Sedate & $\begin{array}{l}\text { Distance } \\
{[\mathrm{mm}]^{\mathrm{b}}}\end{array}$ & Matching \\
\hline 1 & 14 & $\mathrm{R}$ & M & CD (L-TL) & PET & 0.5 & Yes & 3.8 & Yes \\
\hline 2 & 4 & $\mathrm{R}$ & M & CD (R-FL) & PET & 2 & Yes & 44.8 & Yes \\
\hline 3 & 10 & $\mathrm{R}$ & $\mathrm{F}$ & CD (L-TL) & PET & 24 & No & 7.6 & Yes \\
\hline 4 & 8 & $\mathrm{~L}$ & $\mathrm{~F}$ & CD (L-INS) & SPECT & 7 & No & 13.7 & Yes \\
\hline 5 & 15 & $\mathrm{~L}$ & M & TS (M-focal) & SPECT & 25 & No & 12.4 & Yes \\
\hline 6 & 17 & $\mathrm{R}$ & $\mathrm{F}$ & VE (R-FL) & SPECT & 48 & No & 20.8 & Yes \\
\hline 7 & 14 & $\mathrm{~L}$ & M & CD (L-OL) & PET & 28 & Yes & 33.1 & Yes \\
\hline 8 & 11 & $\mathrm{R}$ & M & CD (L-FL) & PET & 48 & Yes & 76.0 & No \\
\hline 9 & 2 & $\mathrm{U}$ & $\mathrm{F}$ & CD (L-TL) & PET & 24 & Yes & 50.0 & Yes \\
\hline 10 & 10 & $\mathrm{R}$ & $\mathrm{F}$ & CD (R-TL) & PET & 72 & Yes & 96.1 & No \\
\hline 11 & 15 & $\mathrm{~L}$ & $\mathrm{~F}$ & NL & PET & 72 & No & 93.2 & No \\
\hline 12 & 2 & $\mathrm{U}$ & M & CD (L-FL) & PET & 24 & Yes & 8.6 & Yes \\
\hline 13 & 7 & $\mathrm{R}$ & $\mathrm{F}$ & CD (L-FL) & PET & 0.5 & No & 10.9 & Yes \\
\hline 14 & 6 & $\mathrm{R}$ & M & NL & PET & 24 & Yes & 23.0 & Yes \\
\hline 15 & 10 & $\mathrm{R}$ & $\mathrm{F}$ & CD (R-FL) & SPECT & 18 & No & 21.1 & Yes \\
\hline 16 & 13 & $\mathrm{R}$ & M & CD (L-TL) & PET & 96 & No & 14.5 & Yes \\
\hline 17 & 7 & $\mathrm{R}$ & $\mathrm{F}$ & NL & PET & 24 & No & 17.3 & Yes \\
\hline 18 & 22 & $\mathrm{U}$ & $\mathrm{F}$ & CD (L-PTL) & PET & 10 & Yes & 11.5 & Yes \\
\hline 19 & 6 & A & M & NL & PET & 96 & Yes & 18.3 & Yes \\
\hline 20 & 4 & $\mathrm{R}$ & $\mathrm{F}$ & TS (M-focal) & SPECT & 72 & Yes & 71.5 & No \\
\hline 21 & 8 & $\mathrm{R}$ & M & NL & SPECT & 0.5 & No & 82.4 & No \\
\hline 22 & 10 & $\mathrm{R}$ & M & NL & SPECT & 24 & Yes & 14.7 & Yes \\
\hline 23 & 8 & $\mathrm{R}$ & $\mathrm{F}$ & NL & SPECT & 24 & Yes & 49.9 & Yes \\
\hline 24 & 10 & $\mathrm{R}$ & $\mathrm{F}$ & NL & SPECT & 0.25 & Yes & 29.7 & Yes \\
\hline 25 & 12 & $\mathrm{R}$ & $\mathrm{F}$ & NL & PET & 0.5 & No & 14.3 & Yes \\
\hline 26 & 11 & $\mathrm{R}$ & M & TS (M-foc) & SPECT & 24 & No & 96.0 & No \\
\hline 27 & 3 & $\mathrm{~L}$ & M & CD (R-PL) & PET & 26 & Yes & 37.6 & Yes \\
\hline 28 & 17 & $\mathrm{R}$ & $\mathrm{F}$ & VE (R-FL) & SPECT & 48 & No & 68.0 & No \\
\hline 29 & 6 & $\mathrm{~L}$ & $\mathrm{~F}$ & CD (L-OL) & SPECT & 48 & Yes & 67.1 & No \\
\hline 26 & 11 & $\mathrm{R}$ & M & TS (M-foc) & SPECT & 24 & No & 96.0 & No \\
\hline
\end{tabular}

Conventions: NM Std., nuclear medicine standard; CD, cortical dysplasia; NL, non-lesional; TS, tuberous sclerosis; VE, viral encephalitis; R, right; L, left; TL, temporal lobe; FL, frontal lobe; PL, parietal lobe; OL, occipital lobe; M-focal, multifocal; ${ }^{\mathrm{a}}$ last seizure-to-rsfMRI interval in hours; ${ }^{\mathrm{b}}$ distance between NM Std. and rs-Mean ROIs. 
rs-Mean. One demonstrated regions of interest in the same lobe without overlapping and 3 more showed activation in another lobe of the same hemisphere; 3 patients demonstrated rs-Mean activation on the opposite hemispheres, and a single rs-Mean case showed no asymmetries between the hemispheres.

\subsection{Selected Exemplary Cases}

Three demonstrative cases have been selected from the group of patients with concordant results (Figure 4). The first case is a non lesional case with clear hypometabolism in an interictal PET study; the second case is a patient with tuberous sclerosis showing a high uptake of the radio-pharmaceutical in an ictal SPECT, and the third a case of cortical dysplasia, with associated increased signal on a T2 FLAIR sequence, and hyperperfusion in the ictal SPECT. The rs-Mean results are clearly demonstrative of the seizure foci.

\subsection{Analysis of Failure Cases}

A specific analysis of the cases in which there was a failure for the rs-Mean to detect the epilepsy focus was also conducted to ascertain common features and look for potential correlations with other diagnostic procedures (EEG, Source Localization, or clinical findings). The 8 cases with no convergent findings are summarized in Table 3. Of these 8 cases, 6 had partial concordant results with other modalities of epilepsy focus localization, clinical findings or both (note bold words in Comments column in Table 3).

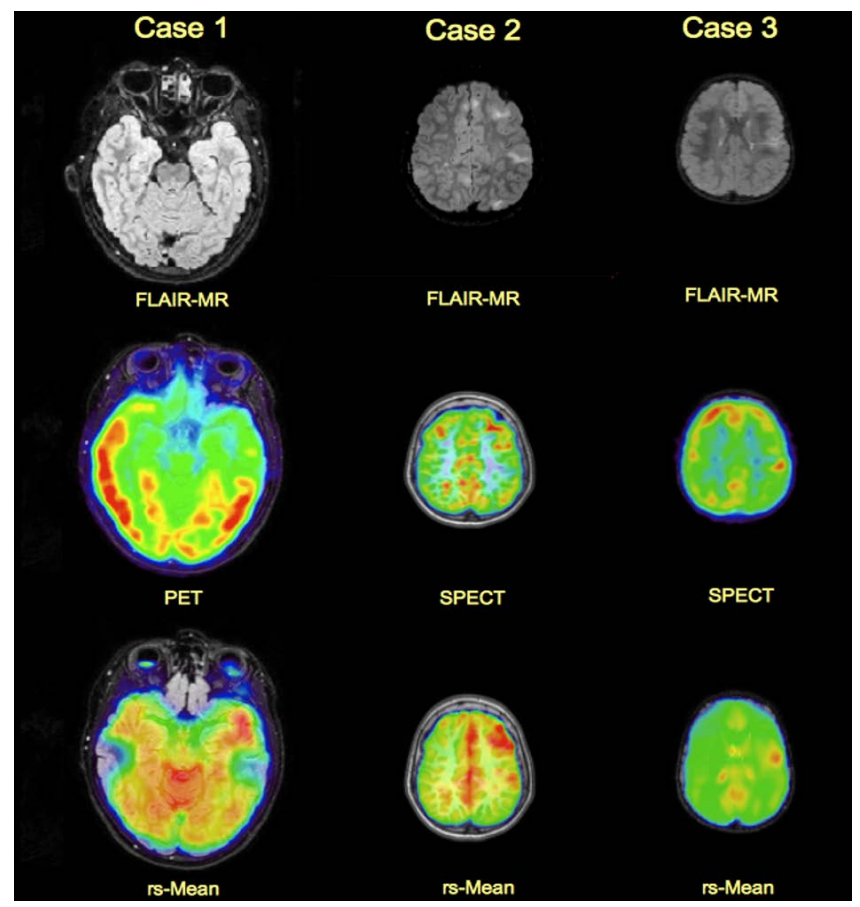

Figure 4. Comparison of results in three selected cases. All insets are in radiological convention (right hemisphere on the left side). The axial cuts of all modalities are located in the same level in each case, chosen accordingly with the best depiction of the area of interest. Column 1. Case with refractory complex partial seizures. No evident lesion was found on MRI. Interictal PET demonstrated a left temporal hypometabolic area in the left temporal lobe (middle inset). The rs-Mean showed high signal coincident with the PET localization (lower inset). Patient had a left temporal resection. Cordical dysplasia was found in pathology. He has remained seizure free since then (June 2012). Column 2. Case: tuberous sclerosis, presenting with motor seizures characterized by head drops, eye-lids twitching and stiffening of the right arm. Structural MRI showed several cortical-subcortical tubers; ictal SPECT showed a left frontal area of slight hyper-perfusion; the rs-Mean showed a well defined high signal area in the same region, coincident with one of the tubers with high interictal EEG activity. Patient has not been operated as of yet. Column 3. Case: motor seizures consisting of unsteadiness and right facial twitching. Structural MRI showed hyperintensity cortical area on FLAIR sequences with focal changes compatible with cortical dysplasia in the left frontal region (upper inset); ictal SPECT shows two areas of hyperperfusion, one related to the left frontal lesion and a second located in the dorsal right frontal area. The rs-Mean showed a clear cluster of high intensity related to the lesion. 
Table 3. Patient specific information and findings.

\begin{tabular}{|c|c|c|c|c|c|c|c|}
\hline Pat \# & Age & Gender & Hand & $\begin{array}{l}\text { rs_Mean } \\
\text { Localiz. }\end{array}$ & $\begin{array}{l}\text { NM Std. } \\
\text { Localiz. }\end{array}$ & $\begin{array}{l}\text { ED } \\
\mathrm{Mm}\end{array}$ & Comments \\
\hline 29 & 6 & F & $\mathrm{LH}$ & L-temporal & L-parietal & 67.1 & $\begin{array}{l}\text { Dx: Cortical Dysplasia. Interictal EEG showed left posterior } \\
\text { quadrant discharges. PET showed complete left hemisphere } \\
\text { hypometabolism. Was not operated. }\end{array}$ \\
\hline 28 & 17 & F & RH & L-temporal & L-parietal & 68.0 & $\begin{array}{l}\text { Dx: Viral Encephalitis. Interictal EEG with left parietal } \\
\text { (P3) spikes. PET showed left central and left temporal } \\
\text { hypometabolism. Was not operated. }\end{array}$ \\
\hline 8 & 11 & M & RH & L-temporal & R-temporal & 76.0 & $\begin{array}{l}\text { Dx: Cortical Dysplasia. Interictal EEG showed independent } \\
\text { bilateral temporal dischargers. Left ones were rare. Patient } \\
\text { had right temporal lobectomy. Asymptomatic since then. }\end{array}$ \\
\hline 10 & 10 & F & RH & $\begin{array}{l}\text { L-frontal } \\
\text { R-mesial- } \\
\text { occipital }\end{array}$ & $\begin{array}{l}\text { R-temporal } \\
\text { R-mesial- } \\
\text { occipital }\end{array}$ & 96.1 & $\begin{array}{l}\text { Dx: Cortical Dysplasia. Two rs-Mean ROIs were found. The } \\
\text { largest ROI was chosen for comparison. Interictal EEG showed } \\
\text { right temporo-occipital discharges. EEG-source localization } \\
\text { showed right-mesial occipital dipole in one solution and the } \\
\text { right inferior posterior temporal in a second solution. Ictal } \\
\text { EEG showed right centro-parietal discharges. rs-Mean } \\
\text { showed evident low signal in the right parietal area. }\end{array}$ \\
\hline 11 & 15 & $\mathrm{~F}$ & LH & L-temporal & $\begin{array}{l}\text { R-temporo- } \\
\text { parietal }\end{array}$ & 93.3 & $\begin{array}{l}\text { Dx: Non lesional. Ictal EEG showed right temporal onset. } \\
\text { rs-Mean has clear low values in the right temporo-parietal } \\
\text { area. Seizure-to-rs-fMRI interval was more than } 72 \text { hours. }\end{array}$ \\
\hline 21 & 8 & M & RH & L-temporal & L-temporal & 82.0 & $\begin{array}{l}\text { Dx: Non lesional. Ictal EEG showed right frontal onset. } \\
\text { The rs-Mean showed evident low signal area in the right } \\
\text { frontal lobe. Seizure-to-rs-fMRI interval was } 1 \text { hour. } \\
\text { The patient underwent right frontal resection. Has } \\
\text { remained seizure free for more than } 12 \text { months. }\end{array}$ \\
\hline 20 & 4 & F & $\mathrm{RH}$ & R-occipital & R-frontal & 71.5 & $\begin{array}{l}\text { Dx: Tuberous Sclerosis. Ictal EEG showed right frontal onset } \\
\text { in majority of registries, but one episode was documented } \\
\text { with "convincing right occipital onset”. The rs-Mean had } \\
\text { clear areas of very low intensities in both frontal lobes } \\
\text { (right more than left). Seizure-to-rs-fMRI interval was } \\
\text { more than } 72 \text { hours. Patient was not operated. }\end{array}$ \\
\hline 26 & 11 & $1 \mathrm{M}$ & RH & L-occipital & R-temporal & 96.0 & $\begin{array}{l}\text { Dx: Tuberous Sclerosis. Ictal and interictal EEG showed } \\
\text { bilateral independent focus in posterior quadrants. Both SPECT } \\
\text { and rs-Mean showed more than one area of hyper intensity. } \\
\text { Comparison was performed between more conspicuous areas. } \\
\text { rs-Mean showed in addition to the left-occipital, left frontal, } \\
\text { right parietal. SPECT findings were subtle. Clinically, the } \\
\text { patient had seizures with right eye and head version to the } \\
\text { right. The patient was not operated. }\end{array}$ \\
\hline
\end{tabular}

Conventions: ED, Euclidian Distance; M, male; F, female; RH, right hand; LH, left hand; L, left; R, right; Dx, diagnosis.

\section{Discussion}

Our results demonstrate that 1 ) resting-state mean volumes in patients with epilepsy differ from normals in intensity and variance, and 2) that abnormal high signal values on the mean volume of a resting state fMRI sequences are correlated with the epileptic focus in a significant number of cases with intractable epilepsy. The explanation of this finding is puzzling. We may consider three possibilities: 1) a localized neural oscillations with higher point of inflection; 2) an interictal-epileptic-discharge-BOLD effect; 3) a T2 local signal increase effect.

\subsection{Localized Network Oscillations}

Normally, the intensity of the spontaneous brain oscillations is small. Usually, the intrinsic networks explain 
only between $1 \%$ and $3 \%$ of the total signal variance within the sequence. Oscillations curves however may have theoretically different points of inflection, producing as a result, different levels of mean signal. An inflection point around 0 , with positive ups and negative downs may cancel out the differences on intensity giving a mean close to 0 . Instead, an inflection point of the oscillation curve located at a higher level will produce a positive value of the mean. The local higher intensity observed in rs-Mean should reflect a local higher BOLD signal during the sequence increasing the inflection point of the oscillations. This explanation, however conflicts with some facts. First, Ad-hoc analysis of the intrinsic networks in each case did not reveal any particular network coinciding with the area shown by the rs-Mean, and second, a higher oscillations in the BOLD response of the epilepsy focus will necessarily be coupled with a relative focal hyperperfusion. However, the rs-fMRI was performed in all cases in an interictal condition, and interictal blood flow is low in the epilepsy focus, as demonstrate by SPECT studies [6] [7].

\subsection{Epileptic Discharge Bold Effect}

Interictal epileptic discharges constitute per-se a spontaneous ongoing endogenous event that triggers bloodflow changes in the affected cortex [8] [9]. Patients with intractable epilepsy, suffer from very frequent epilepsy discharges, and even subclinical electrical seizures preceding overt seizures by several hours [10], that may explain a protracted or sustained local increase of blood flow. However, interictal metabolism and blood flow are low. For example, the first demonstrative case shows high rs-Mean in the same place where the PET study shows less metabolism (rs-Mean and PET were not performed simultaneously but both were performed in an interictal state). Therefore, it is quite difficult to validate that the cause of the high signal in the rs-Mean is explained by the relative higher local BOLD signal.

The third possibility is that the rs-Mean high signal is related to the same higher local T2 signal that has been found in diffusion-weighted MRI associated with convulsive and non-convulsive status epilepticus and tonicclonic generalized seizures [11] [12]. Changes include focal increase of T2 signal attributed to local edema [13]. However, to our knowledge, interictal or peri-ictal MRI transient changes have not been described as of yet. It may be quite possible that frequent or recent interictal discharges increase T2 in a minute scale not detectable in normal sequences but made overt by averaging, color coding or both. These T2 changes would be not oscillatory. In this case the effect would be completely related to tissular and not to vascular changes. Of note is the lack of relationship between the structural hyperintensities observed in the MRI FLAIR images and the rs-Mean signal. Tuberous sclerosis cases show several hyperintensities in FLAIR sequences associated to the tuber localization However, rs-Mean reveals distinctly the intensity of such tubers as demonstrated in Case 2 (see Figure 4) suggesting a more "functional" explanation for the presence of the rs-Mean intensity.

Our work is pioneering in the utilization of the rs-Mean. Previous attempts to correlate rs-fMRI with epilepsy focus activity have been reported [14], but they relate epilepsy focus with connectivity changes. This is the first time the mean BOLD of the resting state condition is utilized for this purpose.

Our work has some limitations due in part to our dedicated clinical environment. First, our comparison group of typical controls does not perfectly match our patient group. Our patients are children between 2 and 22 years of age, while the volunteer group age range is from 22 to 34 . The decision to utilize this sample of typical controls instead of a group of normal volunteers of matching ages was taken favoring number versus matching, since we only had 5 cases of normal children undergoing resting state, associated with a different study. Post-hoc observation of these cases did not reveal any significant difference with the results reported here in the control group. Still, the number of normal subjects is low, but given the small variance observed in the sample, the effect should not be significant.

Second, intensities between the groups may differ because of the inherent effect of the magnets per se. This variable was considered. The authors feel that the normalization of the data to the maximum maxima across the groups occurring in the CSF may control this effect. The CSF may be considered an invariant as it is not influenced by neural oscillations, vascular changes, or seizures.

Third, since many of our patients have daily seizures, lack of EEG validation of the actual electrical condition during the rs-fMRI is problematic. We cannot rule out that some may have actual electrical ictal discharges. However, all patients had the resting-state sequence done as part of a complete protocol for epilepsy workup that includes task-related fMRI or fMRI under sedation with passive paradigms. In all cases, there is a prior evaluation immediately before the fMRI on the behavioral and cognitive status of the patient. Patients with ictal or pe- 
ri-ictal conditions are put off to later scan. All, but patients having several seizures a day, had the fMRI session at least 24 hours after the last clinical seizure.

Of note is the time when the resting-state fMRI took place within the exam session. All subjects had the resting state fMRI done after the tasks of the standard fMRI (all block designed). Since these tasks have at least two language tasks, we cannot discard that some remnant effect is carry over to the rs-Mean in those cases in which it pointed to the left temporal lobe. However, this effect if it actually exists should be negligible. Indeed, we conducted an experiment in a normal volunteer who had initially a basal rs-fMRI. Immediately afterwards the volunteer was asked to squeeze a sponge for 15 continuous minutes and had a second rs-fMRI immediately following the task. No significant differences were found between the intensity in the motor areas between the baseline rs-fMRI and post-task rs-fMRI neither appear noticeable asymmetries in the motor or sensory intrinsic networks.

The importance of this tool is not only attributed to its inherent non-invasive nature and its simplicity, but more importantly to its high availability. Magnetic Resonance machines are nowadays an ubiquitous and indispensable tool, available even in remote and impoverished locations. SPECT or PET machines are less likely to be found in medical facilities and they require immediate availability of the radiopharmaceutical which has a short life, is very costlyand require very specialized support teams and know-how.

\section{Conclusion}

In conclusion, we introduce here for the first time a simple post-processing procedure to localize an epilepsy focus based on rs-fMRI. This novel procedure utilizes a single volume automatically generated, which averages all signal fluctuations in a resting-state fMRI sequence, and seems to render higher intensities in the areas of interictal epileptic activity.

\section{Acknowledgements}

We express our sincere thanks to Jianbin Zhu Ph.D., for his advice on the statistical analysis, and Dr. Michael S. Duchowny, M.D. for the review of the manuscript and his valuable input. The authors declare not to have financial interest in the matters of this publication.

\section{References}

[1] Bellec, P., Rosa-Neto, P., Lyttelton, O.C., Benali, H. and Evans, A.C. (2010) Multi-Level Bootstrap Analysis of Stable Clusters in Resting-State fMRI. NeuroImage, 51, 1126-1139. http://dx.doi.org/10.1016/j.neuroimage.2010.02.082

[2] Calhoun, V.D. and Adal1, T. (2012) Multisubject Independent Component Analysis of fMRI: A Decade of Intrinsic Networks, Default Mode, and Neurodiagnostic Discovery. IEEE Reviews in Biomedical Engineering, 5, 60-73.

[3] Uddin, L.Q., Supekar, K. and Menon, V. (2010) Typical and Atypical Development of Functional Human Brain Networks: Insights from Resting-State FMRI. Front SystNeurosci, 4, 21. http://dx.doi.org/10.3389/fnsys.2010.00021

[4] Bernal, B. and Altman, N.R. (2003) Evidence-Based Medicine: Neuroimaging of Seizures. Neuroimaging Clinics of North America, 13, 211-224. http://dx.doi.org/10.1016/S1052-5149(03)00019-4

[5] Lemieux, L., Krakow, K. and Fish, D.R. (2001) Comparison of Spike-Triggered Functional MRI BOLD Activation and EEG Dipole Model Localization. NeuroImage, 14, 1097-1104. http://dx.doi.org/10.1006/nimg.2001.0896

[6] Ryding, E., Rosén, I., Elmqvist, D. and Ingvar, D.H. (1998) SPECT Measurements with ${ }^{99 m}$ Tc-HM-PAO in Focal Epilepsy. Journal of Cerebral Blood Flow \& Metabolism, 8, S95-S100. http://dx.doi.org/10.1038/jcbfm.1988.38

[7] Pantano, P., Matteucci, C., di Piero, V., Pozzilli, C., Faedda, M.T., Grasso, M.G., et al. (1991) Quantitative Assessment of Cerebral Blood Flow in Partial Epilepsy Using Xe-133 Inhalation and SPECT. Clinical Nuclear Medicine, 16, 898903. http://dx.doi.org/10.1097/00003072-199112000-00003

[8] Lemieux, L., Laufs, H., Carmichael, D., Paul, J.S., Walker, MC. and Duncan, J.S. (2008) Noncanonical Spike-Related BOLD Responses in Focal Epilepsy. Human Brain Mapping, 29, 329-345. http://dx.doi.org/10.1002/hbm.20389

[9] Yerramsetty, K.M., Neely, B.J., Madihally, S.V. and Gasem, K.A.M. (2010) A Skin Permeability Model of Insulin in the Presence of Chemical Penetration Enhancer. International Journal of Pharmaceutics, 388, 13-23. http://dx.doi.org/10.1016/j.ijpharm.2009.12.028

[10] Litt, B., Esteller, R., Echauz, J., D’Alessandro, M., Shor, R., Henry, T., et al. (2001) Epileptic Seizures May Begin Hours in Advance of Clinical Onset: A Report of Five Patients. Neuron, 30, 51-64. 
http://dx.doi.org/10.1016/S0896-6273(01)00262-8

[11] Bauer, G., Gotwald, T., Dobesberger, J., Embacher, N., Felber, S., Bauer, R., et al. (2006) Transient and Permanent Magnetic Resonance Imaging Abnormalities after Complex Partial Status Epilepticus. Epilepsy \& Behavior, 8, 666671. http://dx.doi.org/10.1016/j.yebeh.2006.01.002

[12] Kramer, R.E., Lüders, H., Lesser, R.P., Weinstein, M.R., Dinner, D.S., Morris, H.H., et al. (1987) Transient Focal Abnormalities of Neuroimaging Studies during Focal Status Epilepticus. Epilepsia, 28, 528-532. http://dx.doi.org/10.1111/j.1528-1157.1987.tb03683.x

[13] Kramer, R.E., Lüders, H., Lesser, R.P., Weinstein, M.R., Dinner, D.S., Morris, H.H., et al. (1987) Transient Focal Abnormalities of Neuroimaging Studies during Focal Status Epilepticus. Epilepsia, 28, 528-532. http://dx.doi.org/10.1111/j.1528-1157.1987.tb03683.x

[14] Wurina, Zang, Y.-F. and Zhao, S.-G. (2012) Resting-State fMRI Studies in Epilepsy. Neuroscience Bulletin, 28, 449455. http://dx.doi.org/10.1007/s12264-012-1255-1 